\title{
Coronary Artery Disease and High Altitude: Unresolved Issues
}

\author{
Ivano Bonadei, ${ }^{1}$ Edoardo Sciatti, ${ }^{1,{ }^{*}}$ Enrico Vizzardi, ${ }^{1}$ Marialma Berlendis, ${ }^{1,2}$ Giordano Bozzola, ${ }^{1,2}$ and \\ Marco Metra ${ }^{1}$ \\ ${ }^{1}$ Section of Cardiovascular Disease, Department of Medical and Surgical Specialties, Radiological Sciences and Public Health, University of Brescia, Brescia, Italy \\ ${ }^{2}$ Pneumology Unit, Cardio-Thoracic Department, Spedali Civili od Brescia, Brescia, Italy \\ "Corresponding author: Edoardo Sciatti, Section of Cardiovascular Disease, Department of Medical and Surgical Specialties, Radiological Sciences and Public Health, University \\ of Brescia, Piazzale Spedali Civili 1, 25123 Brescia, Italy. Tel: +39-0303995536, Fax: +39-0303995013, E-mail: edoardo.sc@tin.it
}

Received 2015 August 23; Accepted 2015 September 05.

Keywords: Altitude, Cardiopulmonary Exercise Test, Coronary Artery Disease, Mountaineering

\section{Dear Editor,}

The progressive exposure to an increasing altitude results in a series of adjustments of various organs and systems (i.e., acclimatization) in healthy subjects; this requires them to stay on the mountain for longer or shorter periods. Knowledge about the cardiovascular adaptations to altitude in healthy subjects and known cardiac patients is very important when it comes to recommending appropriate preventive strategies and to assess the risk of adverse events specific to the mountains ("mountain sickness") or pre-existing heart disease. To date, no guidelines concerning cardiac patients and high altitude are available.

Physiology of the cardiopulmonary system at high altitudes: According to Rimoldi and co-workers (1), high altitude can be defined as the terrestrial elevation at which the oxygen hemoglobin saturation decreases below 90\%. At a moderate latitude, this corresponds to an altitude of about $2,500 \mathrm{~m}$. From this altitude and above, hypoxemia triggers a series of pulmonary and cardiovascular adjustments intended to maintain the organism's adequate oxygenation (1); such adjustments include increased pulmonary ventilation and cardiac output, with the latter almost exclusively due to increased heart rate (2-4).

In the heart, the major adjustments are increases in heart rate, cardiac contractility, and cardiac output (5). As a direct consequence of these adjustments, myocardial workload and oxygen demand increase. To respond to this phenomenon, the myocardium has to rely almost exclusively on coronary vasodilatation and enhancement of coronary blood flow because the coronary oxygen extraction is already high at low altitudes (5). In the vessels, the main initial adaptive mechanisms related to altitudeinduced hypoxemia are pulmonary artery vasoconstriction and peripheral and cerebral artery vasodilatation (1).

The stimulation of the cardiovascular system reaches its maximum effects during the first few days of exposure; thereafter, probably due to the beneficial effects of subsequent respiratory, hematological, and muscular adaptation mechanisms, a new steady state is established (1-6). After several days of acclimatization, cardiac output returns to normal values, but the heart rate remains increased, so that the stroke volume is decreased. Left ventricular function is maintained, with initially preserved or slightly depressed indices of systolic function and an altered diastolic filling pattern $(5,6)$. Finally, peripheral vasodilation is opposed by increased sympathetic activity and reduced tissue hypoxia due to acclimatization, resulting in elevated blood pressure (6).

Coronary artery disease and high altitudes: Altitude exposure carries no identified risk of myocardial ischemia in healthy subjects, but it might lead to potential severe consequences in patients with previous cardiovascular conditions (5). Few studies exist on the effects, in terms of safety, of exposure to high altitudes in patients with stable coronary artery disease (CAD). However, some studies have debated the safety of traveling to high altitudes after coronary bypass surgery $(7,8)$.

Some studies have evaluated the coronary reserve that is the difference between coronary perfusion at rest and during maximal vasodilation, in both healthy subjects and those with CAD. Wyss et al. (9) compared the coronary reserve during exercise in the supine position in 10 healthy subjects subjected to inhalation of a hypoxic mixture corresponding to an altitude of 4,500 $\mathrm{m}$ and in 8 CAD patients with significant coronary stenosis at a simulated altitude of 2,500 m using the same method. In healthy subjects, coronary flow was significantly increased compared with that recorded at sea level and the coronary reserve was preserved, although the workload achieved was lower. In contrast, in CAD patients, both coronary flow to the maximum stress and the coronary reserve were reduced com- 
pared to the measured values at sea level (9). This could be explained in relation to impaired endothelial vasomotor control and the additional vasoconstrictive role of alkalosis caused by increased ventilation and sympathetic activity $(1,2,10)$. Whether this phenomenon is of clinical relevance is still debated.

Exercise testing during hypoxia in stable patients with CAD showed little ST deviation, at least if ischemia were absent at low to moderate exercise at low altitudes (11). Schmid et al. (12) studied 15 patients after ST-elevation myocardial infarction (STEMI) and 7 patients after Non-STelevation myocardial infarction (12 months after the acute event) using a maximal, symptom-limited exercise stress test at $500 \mathrm{~m}$ and after a rapid ascent to the Jungfraujoch, Switzerland $(3,454 \mathrm{~m})$. After the maximal exercise stress test, no signs of myocardial ischemia or significant arrhythmias were noted on electrcardiogram (ECG). The authors concluded that rapid ascent and submaximal exercise can be considered safe for low-risk patients 6 months after revascularization for an acute coronary event and a normal exercise stress test at low altitude. Thus, the heart rates and systolic blood pressure products did not differ and the same lactate levels were achieved. In elderly patients, Levine et al. (13) observed that acute exposure to $2,500 \mathrm{~m}$ after an ascent from sea level depressed the ischemic threshold by $5 \%$ on day 1 but not on day 5 .

However, a smaller study showed ST deviations at a similar product between heart rate and systolic blood pressure at a low altitude (14).

del Pilar-Valla et al. (15) showed that intermittent exposure to hypoxia may reduce the ischemia score in patients with stable CAD. These subjects had $4 \mathrm{~h}$ of exposure to hypoxia once a week starting at a simulated altitude of 2,400 $\mathrm{m}$ and a weekly increase of $300 \mathrm{~m}$ (total 14 sessions).

de Vries et al. (16) compared healthy subjects and patients with a history of CAD with a low risk profile and sufficient exercise capacity. They showed that cases and controls did not respond differently to the maximum exercise test with a cycle ergometer at sea level and base camp after 10 days of acclimatization at an altitude of $4,200 \mathrm{~m}$. They suggested that a selected group of patients would tolerate remaining and exercising at high altitude.

Practical considerations and possible recommendations: Considering the heterogeneity of the few available studies, we must acknowledge that it is still impossible to recognize an altitude cut-off under which mountain journeying and/or mountaineering is completely considered safe for stable CAD patients. Moreover, altitude tolerance depends on the severity of the disease, the strength of adaptive mechanisms, and the physical activity associated with the exposure. In contrast, high-altitude exposure may unpredictably determine acute coronary syndrome or worsening angina symptoms, and no studies exist regarding unstable patients. Nevertheless, up to 2,500 m, environmental conditions are similar to those in a commercial aircraft and may be considered safe (6). In addition, asymptomatic or mildly symptomatic stable CAD patients with a negative exercise test at sea level, well-controlled blood pressure, and absence of pulmonary disease can ascend to 3,500 $\mathrm{m}$ with minimal additional risk (6). In every case, a slow ascent allowing acclimatization is mandatory. Indeed, the danger of adverse events is greatest during the first days of acute exposure and may decrease within a few days as oxygen delivery increases. Exercise must be kept to a minimum in the first days of the stay. Finally, for patients desiring to go to extremely high altitudes $(>4,000 \mathrm{~m}$ ) we advise the use of a cardiopulmonary exercise test where they breathe a hypoxic gas mixture, as previously reported by our group (17). This is a simulation of exercise in hypoxic conditions and could give important information about acute cardiopulmonary adaptations to such an adverse environment. We recommend the counseling of a tertiary referral center with the presence of cardiologists and pneumologists specialized in high mountain medicine. Further studies are required before such an approach can be globally recommended.

\section{References}

1. Rimoldi SF, Sartori C, Seiler C, Delacretaz E, Mattle HP, Scherrer U, et al. High-altitude exposure in patients with cardiovascular disease: risk assessment and practical recommendations. Prog Cardiovasc Dis. 2010;52(6):512-24. doi: 10.1016/j.pcad.2010.03.005. [PubMed: 20417345].

2. West JB, Schoene RB, Luks AM, Milledge JS. In: High altitude medicine and physiology. 4 ed. London: Hodder Arnold; 2007. pp. 181-3.Preexisting medical conditions at altitude.

3. Stenberg J, Ekblom B, Messin R. Hemodynamic response to work at simulated altitude, 4,000 m. J Appl Physiol. 1966;21(5):1589-94. [PubMed: 5923231].

4. Vogel JA, Harris CW. Cardiopulmonary responses of resting man during early exposure to high altitude. J Appl Physiol. 1967;22(6):1124-8. [PubMed: 5338456].

5. Naeije R. Physiological adaptation of the cardiovascular system to high altitude. Prog Cardiovasc Dis. 2010;52(6):456-66. doi: 10.1016/j.pcad.2010.03.004. [PubMed: 20417339].

6. Bartsch P, Gibbs JS. Effect of altitude on the heart and the lungs. Circulation. 2007;116(19):2191-202. doi: 10.1161/CIRCULATIONAHA.106.650796. [PubMed: 17984389].

7. Berner G, Froelicher VF, West JB. Trekking in Nepal: safety after coronary artery bypass. JAMA. 1988;259(21):3184.

8. Hultgren HN. The safety of trekking at high altitude after coronary bypass surgery. JAMA. 1988;260(15):2218-9. [PubMed: 3262779].

9. Wyss CA, Koepfli P, Fretz G, Seebauer M, Schirlo C, Kaufmann PA. Influence of altitude exposure on coronary flow reserve. Circulation. 2003;108(10):1202-7. doi: 10.1161/01.CIR.0000087432.63671.2E. [PubMed: 12939217].

10. Mieske K, Flaherty G, O’Brien T. Journeys to high altitude-risks and recommendations for travelers with preexisting medical conditions. 
J Travel Med. 2010;17(1):48-62. doi: 10.1111/j.1708-8305.2009.00369.x. [PubMed: 20074100].

11. Brunner-La Rocca HP. High altitude in the heart of healthy and sick. Cardiovasc Med. 2012;15:41-7.

12. Schmid JP, Noveanu M, Gaillet R, Hellige G, Wahl A, Saner H. Safety and exercise tolerance of acute high altitude exposure (3454 m) among patients with coronary artery disease. Heart. 2006;92(7):921-5. doi: 10.1136/hrt.2005.072520. [PubMed: 16339809].

13. Levine BD, Zuckerman JH, deFilippi CR. Effect of high-altitude exposure in the elderly: the Tenth Mountain Division study. Circulation. 1997;96(4):1224-32. [PubMed: 9286953].

14. Dehnert C, Bartsch P. Can patients with coronary heart disease go to high altitude?. High Alt Med Biol. 2010;11(3):183-8. doi 10.1089/ham.2010.1024. [PubMed: 20919884].
15. del Pilar Valle M, Garcia-Godos F, Woolcott OO, Marticorena JM, Rodriguez V, Gutierrez I, et al. Improvement of myocardial perfusion in coronary patients after intermittent hypobaric hypoxia. J Nucl Cardiol. 2006;13(1):69-74. doi: 10.1016/j.nuclcard.2005.11.008. [PubMed: 16464719].

16. de Vries ST, Kleijn SA, van 't Hof AW, Snaak H, van Enst GC, Kamp O, et al. Impact of high altitude on echocardiographically determined cardiac morphology and function in patients with coronary artery disease and healthy controls. Eur J Echocardiogr. 2010;11(5):446-50. doi: 10.1093/ejechocard/jep237. [PubMed: 20139441].

17. Vizzardi E, Berlendis M, Sciatti E, Bonadei I, Quinzani F, Tassi G, et al. Risk assessment for a high-altitude alpinist with coronary artery disease. Heart Lung Vessel. 2015;7(3):268-70. [PubMed: 26495276]. 